\title{
Study of Quarkonia and Quarkonium-like States at Belle
}

\author{
Sen Jia* \\ Beihang University, the Belle Collaboration \\ E-mail: jiasen@buaa.edu.cn
}

\begin{abstract}
We review recent results on quarkonia and quarkonium-like states from the Belle experiment. An enhancement of the transition $\Upsilon(4 S) \rightarrow \eta \Upsilon(1 S)$ with respect to the transition $\Upsilon(4 S) \rightarrow$ $\pi^{+} \pi^{-} \Upsilon(1 S)$ is confirmed. The first observation of the processes $e^{+} e^{-} \rightarrow \eta \Upsilon_{J}(1 D)$ in $e^{+} e^{-}$ annihilation near $\Upsilon(5 S)$ and $\Upsilon(4 S) \rightarrow \eta^{\prime} \Upsilon(1 S)$ is reported. The new state $X^{*}(3860)$ is clearly observed with a significance of $6.5 \sigma$ in the distribution of invariant mass of $D \bar{D}$. We search for charmonium-like states, including $X(3872)$ in the missing mass spectrum of $K^{+}, Y(4660)$ and its spin partner in the distribution of $\Lambda_{c}^{+} \Lambda_{c}^{-}$invariant mass, and double $Z_{c}$ in $\Upsilon(1 S, 2 S)$ decays and $e^{+} e^{-}$annihilation at $\sqrt{s}=10.52,10.58$, and $10.867 \mathrm{GeV}$. No significant signals are observed in any of the studied modes, and the $90 \%$ confidence level (C.L.) upper limits on their branching fractions or the Born cross sections are determined.
\end{abstract}

XXVI International Workshop on Deep-Inelastic Scattering and Related Subjects (DIS2018) 16-20 April 2018

Kobe, Japan

${ }^{*}$ Speaker. 


\section{Introduction}

The total integrated luminosity of B factories reached $1040 \mathrm{fb}^{-1}$ [1]. Considerable research efforts have been devoted to the study of quarkonia and quarkonium-like States using rich data samples taken in the $\Upsilon(1 S, 2 S, 3 S, 4 S, 5 S)$ resonances. Bottomonium spectroscopy was gradually developed by the study of transitions between its states. The charmonium-like states have been intensively investigated in the $B$ decays, and initial state radiation (ISR) and two-photon processes at Belle. The bottomonium-like states, including $Z_{b}(10610)$ and $Z_{b}(10650)$ are now quite well known objects thanks to Belle studies. In this review, the transitions between bottomonium states and the search for some charmonium-like states, e.g., $X(3872), Y(4660)$, etc, are mainly reported.

\section{The study of quarkonia at Belle}

\subsection{The hadronic transition in bottomonium decay}

The transitions between bottomonium states via an $\eta$ meson are predicted in Refs. [2, 3, 4], to be highly suppressed, since they require a spin flip of the heavy quark. However, in experiment, the results are conflicted with those from theoretical predictions. Belle [5] measured the branching fractions $\mathscr{B}\left(\Upsilon(4 S) \rightarrow \pi^{+} \pi^{-} \Upsilon(1 S)\right)$ and $\mathscr{B}(\Upsilon(4 S) \rightarrow \eta \Upsilon(1 S))$, and gave the ratio of branching fractions:

$$
\mathscr{R}=\frac{\mathscr{B}(\Upsilon(4 S) \rightarrow \eta \Upsilon(1 S))}{\mathscr{B}\left(\Upsilon(4 S) \rightarrow \pi^{+} \pi^{-} \Upsilon(1 S)\right)}=2.07 \pm 0.30 \pm 0.11,
$$

where the first uncertainty is statistical, while the second is systematic. These results are consistent with the prior measurements by BABAR [6] within a reasonable error range. They confirmed an enhancement of the transition $\Upsilon(4 S) \rightarrow \eta \Upsilon(1 S)$ with respect to the transition via a dipion. Given the possible $\Upsilon\left(1^{3} D_{1,2}\right)$ production through double-radiative transitions from the $\Upsilon(4 S)$ through the $\chi_{b J}(2 P)$, the search for the $\Upsilon\left(1^{3} D_{1,2}\right)$ were also performed in Ref. [5]. No significant signals are observed, and the upper limits at $90 \%$ C.L. on the product of branching fractions are obtained to be $\mathscr{B}\left(\Upsilon(4 S) \rightarrow \gamma \gamma \Upsilon\left(1^{3} D_{1,2}\right)\right) \times \mathscr{B}\left(\Upsilon\left(1^{3} D_{1,2}\right) \rightarrow \eta \Upsilon(1 S)\right)<2.3 \times 10^{-5}$.

The $\eta$ transition in higer mass bottomonium spectroscopy, e.g., $e^{+} e^{-}$reaction near the $\Upsilon(5 S)$, was studied by Belle [7] as well. In this analysis, only the $\eta$ is reconstructed by $\gamma \gamma$ and the recoil mass spectrum of $\eta$ candidates is investigated. The clear $\Upsilon(1 D)$ signal could be observed, and the evidence for the $e^{+} e^{-} \rightarrow \eta \Upsilon(2 S)$ transition was reported. However, no hints are found for the signals of $\Upsilon(1 S), h_{b}(1 P)$ and $h_{b}(2 P)$. In particular, the branching fraction of $e^{+} e^{-} \rightarrow \eta \Upsilon(1 D)$ is compatible with the prediction via hadronic loop machanism in Ref. [8]. No direct evidence is shown to prove the presence of the three states of the $\Upsilon(1 D)$ triplet, which attribute to the limited experimental resolution and the possible mixture of different states of the $\Upsilon(1 D)$ triplet.

The kinematically allowed transition $\Upsilon(4 S) \rightarrow \eta^{\prime} \Upsilon(1 S)$ is excepted to be similarly enhanced as $\Upsilon(4 S) \rightarrow \eta \Upsilon(1 S)$ [9]. Belle [10] searched for the the hadronic transition $\Upsilon(4 S) \rightarrow \eta^{\prime} \Upsilon(1 S)$ using $496 \mathrm{fb}^{-1}$ data collected at the $\Upsilon(4 S)$ resonance. The $\eta^{\prime}$ is reconstructed by $\rho^{0} \gamma(2 \pi 1 \gamma)$ and $\pi^{+} \pi^{-} \eta(2 \pi 2 \gamma)$, with $\eta \rightarrow \gamma \gamma$. Figure 1 shows the $\Delta M_{\eta^{\prime}}\left(M(\Upsilon(4 S))-M(\Upsilon(1 S))-M\left(\eta^{\prime}\right)\right)$ distribution in mode $2 \pi 1 \gamma$ and $2 \pi 2 \gamma$. From Fig. 1, the clear signals for the transition $\Upsilon(4 S) \rightarrow$ $\eta^{\prime} \Upsilon(1 S)$ could be observed. The statistical significance of the combined measurement is $5.7 \sigma$ after considering yield-related systematic uncertainties. Finally, the measured branching fraction 
is $\mathscr{B}\left(\Upsilon(4 S) \rightarrow \eta^{\prime} \Upsilon(1 S)\right)=(3.43 \pm 0.88$ (stat. $) \pm 0.21$ (syst. $\left.)\right) \times 10^{-5}$. The first observation of an $\eta^{\prime}$ transition between bottomonia present a wide range of possibilities for the further development of bottomonium spectroscopy.
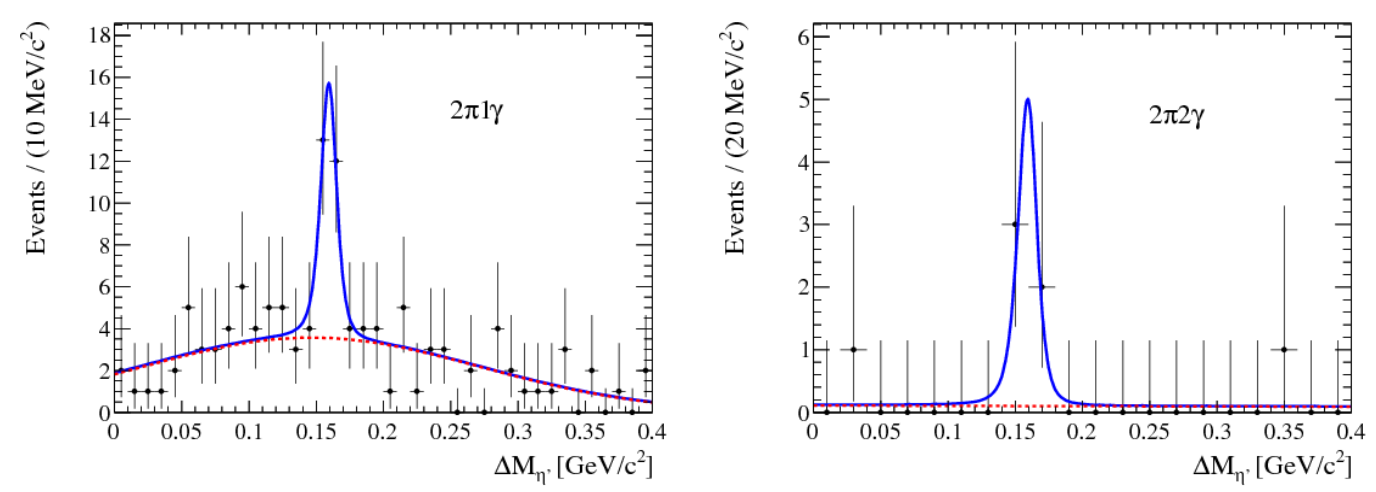

Figure 1: Fit to the $\Delta M_{\eta^{\prime}}$ distribution for $\Upsilon(4 S) \rightarrow \eta^{\prime} \Upsilon(1 S)$ candidates reconstructed in the $2 \pi 1 \gamma$ (left) and $2 \pi 2 \gamma$ (right) final states. Data are shown as points, the solid blue line shows the best fit to the data, while the dashed red line shows the background contribution.

\subsection{An alternative $\chi_{c 0}(2 P)$ candidate in $e^{+} e^{-} \rightarrow J / \psi D \bar{D}$}

The $\chi_{c 2}(2 P)$ candidate was observed by Belle [11] and BABAR [12] in the process $\gamma \gamma \rightarrow D \bar{D}$. In Ref. [13], the authors show that there is an indication of the $\chi_{c 0}(2 P)$ with a mass around 3840 $\mathrm{MeV}$ and width of about $200 \mathrm{MeV}$ with combined Belle [11] and BABAR [12] data. However, the parameters measured in Ref. [13] are biased, e.g., the $\chi_{c 0}(2 P)$ mass is shifted to lower values [16]. To search $\chi_{c 0}(2 P)$ candidate and measure its parameters, Belle [14] presented an updated analysis of the process $e^{+} e^{-} \rightarrow J / \psi D \bar{D}$ using 1.4 times luminosity greater than the previous analysis [15]. A multivariate method is applied to improve the discrimination of the signal and background events. The distribution of invariant mass of $D \bar{D}$ is shown in Fig. 2, where the new state $X^{*}(3860)$ is clearly observed with a significance of $6.5 \sigma$. An amplitude analysis is performed to study the $J^{P C}$ quantum numbers of the $D \bar{D}$ system. In the default model (constant nonresonant amplitude), exclusion levels of the $2^{++}$hypothesis is found to be $3.8 \sigma$, and confidence levels of the $2^{++}$hypothesis is up to $77 \%$.

\subsection{Bottomonium inclusive decays}

The $\chi_{c 1}$ and $f_{1}(1285)$ productions in $\Upsilon(1 S, 2 S)$ inclusive decays were measured by Belle [17, 18]. The differences in the MC-determined reconstruction efficiencies for different $\chi_{c 1}$ and $f_{1}(1285)$ momenta have been considered by partitioning the data samples according to the scaled momentum of $\chi_{c 1}$ and $f_{1}(1285)$ in $e^{+} e^{-}$center-of-mass (C.M.) frame. The inclusive branching fractions $\mathscr{B}\left(\Upsilon(1 S) \rightarrow \chi_{c 1}+\right.$ anything $)=(1.90 \pm 0.43$ (stat. $) \pm 0.14$ (syst. $\left.)\right) \times 10^{-4}$ is measured with an improved precision over prior measurements [19], and $\mathscr{B}\left(\Upsilon(2 S) \rightarrow \chi_{c 1}+\right.$ anything $)=$ $(2.24 \pm 0.44$ (stat. $) \pm 0.20($ syst. $)) \times 10^{-4}, \mathscr{B}\left(\Upsilon(1 S) \rightarrow f_{1}(1285)+\right.$ anything $)=(46 \pm 28$ (stat. $) \pm$ $13($ syst. $)) \times 10^{-4}$ and $\mathscr{B}\left(\Upsilon(2 S) \rightarrow f_{1}(1285)+\right.$ anything $)=(22 \pm 15($ stat.$) \pm 63($ syst. $)) \times 10^{-4}$ are measured for the first time. 


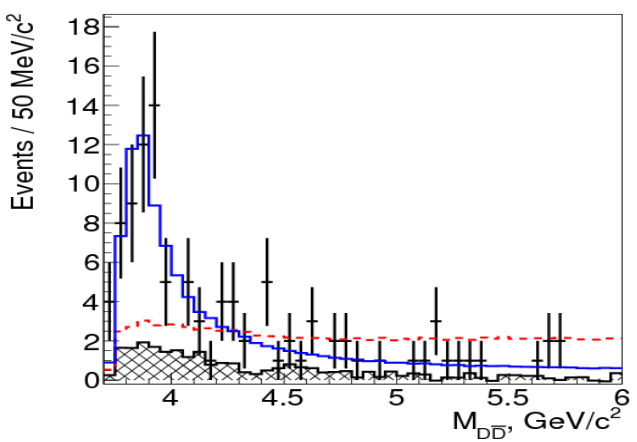

Figure 2: The invariant mass spectrum of $D \bar{D}$ in the $e^{+} e^{-} \rightarrow J / \psi D \bar{D}$. The points with error bars are the data, the hatched histogram is the background, the blue solid line is the fit with a new resonance $X^{*}(3860)$ $\left(J^{P C}=0^{++}\right)$and the red dashed line is the fit with nonresonant amplitude only.

Belle [18] performed the measurement of $\chi_{b J} \rightarrow J / \psi+$ anything, where the $\chi_{b J}$ is identified through the decay $\Upsilon(2 S) \rightarrow \gamma \chi_{b J}$. As shown in Fig. 3 of the spectrum of the $\Upsilon(2 S)$ radiative photon energy in the $e^{+} e^{-}$C.M. frame, a clear $\chi_{b 2}$ signal may be observed with the statistical significances of $3.5 \sigma$. For the first time, the $\chi_{b 2} \rightarrow J / \psi+$ anything branching fraction was reported to be $(1.50 \pm$ 0.34 (stat.) \pm 0.22 (syst. $)) \times 10^{-3}$. The $90 \%$ C.L. upper limits for the $\chi_{b 0, b 1} \rightarrow J / \psi+$ anything branching fractions are set.

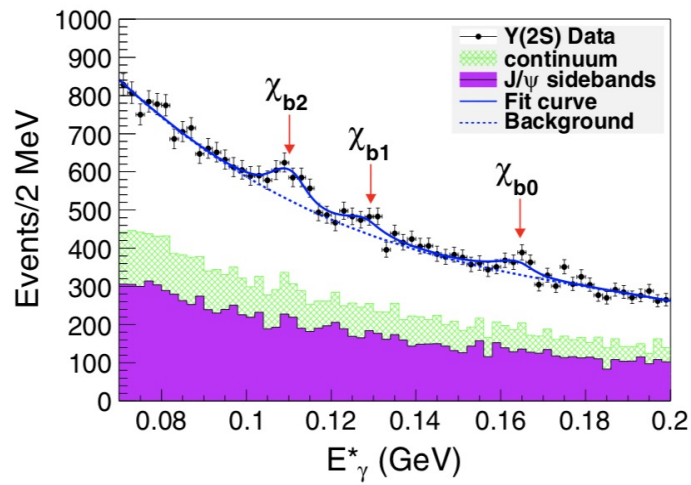

Figure 3: The spectra of the $\Upsilon(2 S)$ radiative photon energy in the $e^{+} e^{-}$C.M. frame in $\Upsilon(2 S)$ data. The dots with error bars are the $\Upsilon(2 S)$ data. The blue solid line is the best fit, and the blue dotted line represents the backgrounds. The magenta shaded histogram is from the normalized $J / \psi$ sideband and the green crosshatched histogram is from the normalized continuum contributions.

\section{The study of quarkonium-like states at Belle}

\subsection{The absolute branching fractions of $B^{+} \rightarrow X_{c \bar{c}} K^{+}$}

The discovery of the $X(3872)$ by the Belle collaboration [20] opened a new era in the field of hadron spectroscopy. Considerable efforts in theory have been devoted to interpret $X(3872)$ as tetraquarks, molecules, hybrids, or hadrocharmonia. It is worthwhile mentioning that a measurement of the absolute branching fraction $B^{+} \rightarrow X(3872) K^{+}$is quite useful. For example, 
$X(3872)[21]$ is regarded as a mixed molecule-charmonium state when $\mathscr{B}\left(B^{+} \rightarrow X(3872) K^{+}\right)=$ $(1.00 \pm 0.68) \times 10^{-5}$.

Recently, Belle [22] presented a measurement of $\mathscr{B}\left(B^{+} \rightarrow X(3872) K^{+}\right)$, along with a simultaneous measurement of the various charmonium(-like) states $\left(X_{c \bar{c}}\right.$ denotes $\eta_{c}, J / \psi, \chi_{c 0}, \chi_{c 1}, \eta_{c}(2 S)$, $\psi(2 S), \psi(3770)$, and $X(3915))$ that appear in the missing mass spectrum. Fully reconstruction for one of the two charged B measons $\left(B_{\text {tag }}\right)$ and NeuroBayes neural-network is used to extract the signals and suppress continuum backgrounds throughout this analysis. The signals are identified at the nominal $X_{c \bar{c}}$ mass in the distribution of missing mass: $M_{\text {miss }}\left(K^{+}\right)=\sqrt{\left(p_{e^{+} e^{-}}^{*}-p_{\text {tag }}^{*}-p_{h}^{*}\right)} / \mathrm{c}$, where $p_{e^{+} e^{-}}^{*}, p_{\text {tag }}^{*}$, and $p_{h}^{*}$ are the four-momenta of the electron-positron initial state, $B_{\text {tag }}$, and $K^{+}$, respectively, in the C.M. frame. No significant signal for $X(3872)$ is observed, and the upper limit at $90 \%$ C.L. on the $\mathscr{B}\left(B^{+} \rightarrow X(3872) K^{+}\right)$is determined to be $2.7 \times 10^{-4}$, which is more stringent than that determined by BaBar [23] $\left(3.2 \times 10^{-4}\right)$. The $90 \%$ C.L. upper limit of $\mathscr{B}\left(B^{+} \rightarrow X(3915) K^{+}\right)=$ $2.9 \times 10^{-4}$ is obtained for the first time. The measured branching fractions for $\eta_{c}$ and $\eta_{c}(2 S)$ are the most precise to date: $\mathscr{B}\left(B^{+} \rightarrow \eta_{c} K^{+}\right)=(12.3 \pm 0.8($ stat. $) \pm 0.7($ syst. $)) \times 10^{-4}$ and $\mathscr{B}\left(B^{+} \rightarrow\right.$ $\left.\eta_{c}(2 S) K^{+}\right)=(4.9 \pm 1.1($ stat.$) \pm 0.3($ syst. $)) \times 10^{-4}$.

\section{2 $Y(4660)$ and its spin partner}

In Refs. [24, 25], the authors predict that the $Y(4660)\left(f_{0}(980) \psi^{\prime}\right.$ bound state) and its spin partner denoted as the $Y_{\eta}\left(f_{0}(980) \eta_{c}(2 S)\right.$ bound state) have a large partial width into $\Lambda_{c}^{+} \Lambda_{c}^{-}$. An updated measurement of $B^{-} \rightarrow K^{-} \Lambda_{c}^{+} \Lambda_{c}^{-}$was performed [26] to search the charmonium-like states $Y(4660)$ and $Y_{\eta}$. No clear $Y(4660)$ and $Y_{\eta}$ signals is evident in the $M\left(\Lambda_{c}^{+} \Lambda_{c}^{-}\right)$spectrum. 90\% C.L. upper limits on $\mathscr{B}\left(B^{-} \rightarrow K^{-} Y\right) \mathscr{B}\left(Y \rightarrow \Lambda_{c}^{+} \Lambda_{c}^{-}\right)$are determined to be $2.0 \times 10^{-4}$ and $1.2 \times 10^{-4}$ for $Y=Y_{\eta}$ and $Y(4660)$, respectively.

\subsection{Double $Z_{c}$ production in $\Upsilon(1 S, 2 S)$ decays and $e^{+} e^{-}$reactions}

In order to understand the nature of $Z_{c}$ state, the measurements of the process $e^{+} e^{-} \rightarrow Z_{c}^{+} Z_{c}^{-}$ is quite useful. For $e^{+} e^{-} \rightarrow Z_{c}^{+} Z_{c}^{-}$, the dependence on $s$ (the $e^{+} e^{-}$C.M. energy squared) of the electromagnetic form factor, $F_{Z_{c}^{+}} Z_{c}^{-}$is $1 / s^{3}$ for a $Z_{c}$ state with tetraquark structure or $1 / s$ for a $Z_{c}$ system of two tightly bound diquarks [27, 28]. Very recently, Belle [29] reported the search for $Z_{c}$ pair production in $\Upsilon(1 S)$ and $\Upsilon(2 S)$ decays as well as in $e^{+} e^{-}$annihilation at $\sqrt{s}=10.52,10.58$, and $10.867 \mathrm{GeV}$. In the search, the decay modes considered are: $Z_{c}^{+}(3900) / Z_{c}^{+}(4200) \rightarrow \pi^{+} J / \psi$, $Z_{c 1}^{+}(4050) / Z_{c 2}^{+}(4250) \rightarrow \pi^{+} \chi_{c 1}(1 P)$ and $Z_{c}^{+}(4050) / Z_{c}^{+}(4430) \rightarrow \pi^{+} \psi(2 S)$. No significant signals are observed in any of the studied modes, and the $90 \%$ C.L. upper limits on their product branching fractions in $\Upsilon(1 S)$ and $\Upsilon(2 S)$ decays and the product of Born cross section and branching fraction for $e^{+} e^{-} \rightarrow Z_{c}^{+} Z_{c}^{(\prime)}{ }^{-}$at $\sqrt{s}=10.52,10.58$, and $10.867 \mathrm{GeV}$ are obtained; $\mathscr{B}\left(\Upsilon(1 S, 2 S) \rightarrow Z_{c}^{+} Z_{c}^{(\prime)-}\right) \times \mathscr{B}\left(Z_{c}^{+} \rightarrow \pi^{+}+c \bar{c}\right)\left(c \bar{c}=J / \psi, \chi_{c 1}(1 P), \psi(2 S)\right)$ within a range of 1 to $50 \times 10^{-6}$ and $\sigma\left(e^{+} e^{-} \rightarrow Z_{c}^{+} Z_{c}^{(\prime)-}\right) \times \mathscr{B}\left(Z_{c}^{+} \rightarrow \pi^{+}+c \bar{c}\right)$ within a range of 0 to $100 \mathrm{fb}^{-1}$.

\section{Summary}

We review some results on the study of quarkonia and quarkonium-like states at Belle. The mainly results are summarized below. (1) the enhancement of the transition $\Upsilon(4 S) \rightarrow \eta \Upsilon(1 S)$ with 
respect to the transition via dipion, (2) the first observation of the process $e^{+} e^{-} \rightarrow \eta \Upsilon_{J}(1 D)$ and $\Upsilon(4 S) \rightarrow \eta^{\prime} \Upsilon(1 S)$, and (3) the $90 \%$ C.L. upper limits are set for the search for some charmoniumlike states, e.g., $X(3872), Y(4660)$ and its spin partner, and $Z_{c}$ pair.

\section{References}

[1] J. Brodzicka et al., Prog. Theor. Exp. Phys. 2012, 04D001 (2012).

[2] Y. P. Kuang, Front. Phys. China 1, 19 (2006).

[3] M. B. Voloshin, Prog. Part. Nucl. Phys. 61, 455 (2008).

[4] Y. A. Simonov and A. I. Veselov, Phys. Lett. B 673, 211 (2009).

[5] E. Guido et al. (Belle Collaboration), Phys. Rev. D 96, 052005 (2017).

[6] B. Aubert et al. (BABAR Collaboration), Phys. Rev. D 78, 112002 (2008).

[7] U. Tamponi et al. (Belle Collaboration), arXiv:1803.03225.

[8] B. Wang, X. Liu, D. Y. Chen, Phys. Rev. D 94, 094039 (2016).

[9] M. B. Voloshin, Mod. Phys. Lett. A 26, 773 (2011).

[10] E. Guido et al. (Belle Collaboration), arXiv:1803.10303.

[11] S. Uehara et al. (Belle Collaboration), Phys. Rev. Lett. 96, 082003 (2006).

[12] B. Aubert et al. (BABAR Collaboration), Phys. Rev. D 81, 092003 (2010).

[13] F. K. Guo and U. G. Meissner, Phys. Rev. D 86, 091501 (2012).

[14] K. Chilikin et al. (Belle Collaboration), Phys. Rev. D 95, 112003 (2017).

[15] P. Pakhlov et al. (Belle Collaboration), Phys. Rev. Lett. 100, 202001 (2008).

[16] S. L. Olsen, Phys. Rev. D 91, 057501 (2015).

[17] S. Jia et al. (Belle Collaboration), Phys. Rev. D 95, 012001 (2017).

[18] S. Jia et al. (Belle Collaboration), Phys. Rev. D 96, 112002 (2017).

[19] R. A. Briere et al. (CLEO Collaboration), Phys. Rev. D 70, 072001 (2004).

[20] S. K. Choi et al. (CLEO Collaboration), Phys. Rev. Lett. 91, 262001 (2003).

[21] C. M. Zanetti, M. Nielsen and R. D. Matheus, Phys. Lett. B 702, 359 (2011).

[22] Y. Kato et al. (CLEO Collaboration), Phys. Rev. D 97, 012005 (2018).

[23] B. Aubert et al. (BABAR Collaboration), Phys. Rev. Lett. 96, 052002 (2006).

[24] F. K. Guo, J. Haidenbauer, C. Hanhart, U.G. Meissner, Phys. Rev. D 82, 094008 (2010).

[25] F. K. Guo, C. Hanhart, U.G. Meissner, Phys. Rev. Lett. 102, 242004 (2009).

[26] Y. B. Li et al. (Belle Collaboration), Eur. Phys. J. C 78, 252 (2018).

[27] S. J. Brodsky et al., Phys. Lett. B 764, 174 (2017).

[28] S. J. Brodsky and R. F. Lebed, Phys. Rev. D 91, 114025 (2015).

[29] S. Jia et al. (Belle Collaboration), arXiv:1805.02308. 